\title{
Effect of restricted spinal motion on gait
}

\author{
Regina Konz, MS; ${ }^{1-2 *}$ Stefania Fatone, PhD; ${ }^{1,3}$ Steven Gard, PhD $^{1-4}$ \\ ${ }^{1}$ Northwestern University Prosthetics Research Laboratory and Rehabilitation Engineering Research Program, Chicago, \\ IL; ${ }^{2}$ Department of Biomedical Engineering, Robert R. McCormick School of Engineering and Applied Science, North- \\ western University, Evanston, $I L ;{ }^{3}$ Department of Physical Medicine and Rehabilitation, Feinberg School of Medicine, \\ Northwestern University, Chicago, IL; ${ }^{4}$ Jesse Brown Department of Veterans Affairs Medical Center, Chicago, IL
}

\begin{abstract}
Spinal orthoses are common in the treatment of various conditions that affect the spine. They encompass both the spine and pelvis and thus have implications for pelvic and lowerlimb motion during walking in addition to a direct effect on spinal motion. The role of the spine in walking is largely ill-defined, and the consequences of restricted spinal motion on walking have yet to be explored. This study investigated the effect of spinal restriction on gait in able-bodied persons. Gait analyses were performed on 10 able-bodied subjects as they walked at five different speeds that were distributed across their comfortable range of speeds. Data were collected during walking with and without spinal restriction by a fiberglass body jacket, which is similar to a thoracolumbosacral orthosis (TLSO). With spinal restriction, peak-to-peak (PP) pelvic obliquity and rotation were significantly reduced across all walking speeds $(p<0.001)$, while PP pelvic tilt was significantly reduced at only the fastest walking speeds $(p=0.017)$. PP hip abduction-adduction motion was significantly reduced with spinal restriction across all speeds $(p<$ 0.001 ), while PP hip flexion-extension significantly increased at only the slow and very slow speeds $(p<0.001$ and $p=0.023$, respectively). A better understanding of the effects of restricted spinal motion on gait may help clinicians predict and avoid development of additional problems from TLSO use or surgical restriction of spinal motion. An awareness of these issues will enable clinicians to monitor patients for problems that may result from decreased spine and pelvic motion.
\end{abstract}

Key words: braces, gait, immobilization, locomotion, orthotic devices, spinal curvature, spinal diseases, spinal fusion, spine, walking.

\section{INTRODUCTION}

Spinal motion is believed to play a significant role in maintaining upright posture and balance [1-3] and reducing shock transmission to the head during gait [4]. When spinal deformities impede the spine's ability to compensate for postural changes, trunk imbalance may occur with clinically observable gait deviations. If a deformity exceeds the spine's compensatory abilities, then stabilization, pain relief, and/or correction of spinal deformity may be provided by spinal orthoses, such as a thoracolumbosacral orthosis (TLSO), or surgical instrumentation. Both of these treatment modalities alter alignment and restrict spinal movement.

Spinal orthoses are commonly used in the treatment of various conditions that affect the spine. The objectives of spinal orthoses include control of pain, protection

\footnotetext{
Abbreviations: ANOVA $=$ analysis of variance, ASIS = anterior superior iliac spine, $\mathrm{L}=$ lumbar, MAC = Motion Analysis Corporation, $\mathrm{PP}=$ peak-to-peak, $\mathrm{ROM}$ = range of motion, $\mathrm{S}=$ sacral, $\mathrm{SD}=$ standard deviation, TLSO = thoracolumbosacral orthosis, VACMARL = Department of Veterans Affairs Chicago Motion Analysis Research Laboratory, VGRF = vertical ground reaction force.

* Address correspondence to Regina Konz, MS; Northwestern University Prosthetics Research Laboratory and Rehabilitation Engineering Research Program, 345 East Superior Street, RIC 1441, Chicago, IL 60611; 312-238-6500; fax: 312238-6510. Email: r-konz@northwestern.edu

DOI: 10.1682/JRRD.2004.11.0146
} 
against further injury, assistance for muscle weakness, prevention and/or correction of further deformity, and service as a kinesthetic reminder. Spinal orthoses achieve these objectives primarily by restricting movement of the spine. However, spinal orthoses such as TLSOs encompass both the spine and the pelvis and thus have implications for pelvic and lower-limb motion during walking in addition to a direct effect on spinal motion.

Past studies have examined the effects of spinal orthoses on intervertebral motion [5-7], gross spinal motion [8], and restriction of the spine in positions of maximal trunk flexion-extension-rotation [6-10]. Association of results from these evaluations to the spinal motions that occur during walking is difficult. Waters and Morris measured the effect of orthoses (chairback orthosis and lumbo-sacral corset) on electromyographic activity of the trunk muscles during level walking at different speeds [11]. They found that the corset had little or no effect, while the chairback orthosis increased electromyographic activity of back muscles at fast walking speeds. Nachemson and Elfstrom studied the effect of orthoses (Milwaukee and body cast) on the loads borne by spinal instrumentation (Harrington rods monitored with strain gauges) during supine, sitting, and standing positions and during level walking [12]. Both the Milwaukee orthosis and body cast were found to reduce axial force during relaxed standing and level walking but not in supine or sitting positions. To our knowledge, the effects of spinal orthoses on lower-limb kinematics, pelvic motion, and kinetics during walking have not been previously investigated.

Spinal orthoses should provide spinal stability, pain relief, and normal spinal alignment and balance. An orthosis such as a TLSO, which encompasses the spine as well as the pelvis, has implications for alteration of spinal and pelvic motions. While spinal orthoses may be necessary for the treatment of a given spinal pathology, their use may have consequences for gait of which clinicians should be aware when prescribing and fitting these devices. This study investigated the effect of spinal restriction on gait in able-bodied persons wearing a fiberglass body jacket similar to a TLSO.

We hypothesized that in an otherwise able-bodied person, restriction of spinal motion will result in deviations from "normal" gait patterns in the lower limbs and pelvis. These deviations may occur because of the adoption of compensatory maneuvers or because of coupling between the spine and pelvis and the pelvis and lower limbs. In particular, we believe that since the body jacket encompasses the pelvis as well as the spine, pelvic rotation in all three planes may be altered when a person walks with a restricted spine. These alterations could potentially decrease step length and cadence and thereby affect walking speed. As previously described, spinal motion is believed to play a significant role in maintaining one's balance [1-3]. We hypothesized that subjects will walk slower and increase their base of support (i.e., increase step width) with spinal restriction, since the contribution of spinal motion to maintaining balance will be limited by the body jacket.

Pelvic obliquity and knee flexion during stance have been identified as mechanisms that attenuate shock forces during able-bodied gait [13-14]. Thus, limiting the "normal" motions of the spinal joints and pelvis may directly affect these motions, which may reduce the body's ability to attenuate shock forces. Furthermore, the first peak in the magnitude of the vertical ground reaction force (VGRF) indicates vertical deceleration of the body center of mass and is thought to reflect changes in system compliance associated with shock absorption [15]. Therefore, we postulate that the magnitude of the first peak of the VGRF may increase with spinal restriction during walking because of a potential reduction in shockabsorption ability.

\section{METHODS}

This study was approved by the Northwestern University Institutional Review Board. Ten able-bodied persons (five females and five males) ranging in age from 23 to 37 years (mean \pm standard deviation [SD] $=27 \pm 4$ years) were enrolled. Subjects ranged in height from 160 to $180 \mathrm{~cm}$ (mean $\pm \mathrm{SD}=171 \pm 6 \mathrm{~cm}$ ) and in mass from 54.0 to $97.3 \mathrm{~kg}$ (mean $\pm \mathrm{SD}=72 \pm 12 \mathrm{~kg}$ ). Information about the research subjects is summarized in Table 1. After the experimental protocol was explained to each research subject, we obtained their informed consent. We clinically assessed subjects to ensure that they were "able-bodied" and excluded them if they were found to have a severe leg-length discrepancy, hip-flexion contracture, or history of scoliosis or other spinal anomaly.

The protocol consisted of two successive gait analyses. The first analysis examined each subject's conventional, unrestricted gait. The second analysis examined each subject's gait with the spine restricted by a fiberglass body jacket. During the gait analyses, subjects wore 
Table 1.

Subject data.

\begin{tabular}{|c|c|c|c|c|}
\hline Subject & Sex & $\begin{array}{l}\text { Age } \\
\text { (yr) }\end{array}$ & $\begin{array}{c}\text { Height } \\
\text { (cm) }\end{array}$ & $\begin{array}{c}\text { Mass } \\
\text { (kg) }\end{array}$ \\
\hline 1 & $\mathrm{~F}$ & 26 & 161.5 & 60.5 \\
\hline 2 & $\mathrm{~F}$ & 24 & 166.5 & 75.3 \\
\hline 3 & $\mathrm{~F}$ & 29 & 169.0 & 68.5 \\
\hline 4 & $\mathrm{~F}$ & 24 & 170.0 & 54.0 \\
\hline 5 & $\mathrm{~F}$ & 24 & 160.0 & 65.3 \\
\hline 6 & M & 23 & 179.8 & 82.5 \\
\hline 7 & M & 37 & 178.3 & 82.4 \\
\hline 8 & M & 30 & 172.0 & 72.0 \\
\hline 9 & M & 25 & 173.0 & 61.5 \\
\hline 10 & M & 29 & 177.0 & 97.3 \\
\hline$\overline{\text { Mean } \pm \text { SD }}$ & - & $27.1 \pm 4.3$ & $170.7 \pm 6.4$ & $71.9 \pm 12.2$ \\
\hline
\end{tabular}

shorts and standardized gym shoes for uniformity in footwear. Retroreflective markers were applied directly to the skin with double-sided hypoallergenic tape. The marker set that was used consisted of a modified Helen Hayes arrangement [16] (Figure 1). We used these markers to create a link-segment model of the lower body, which enabled calculation of the kinematic data.

A qualified orthotist custom-formed a fiberglass body jacket to each subject. While the subject stood in a neutral, self-selected position, fiberglass tape was applied circumferentially to his or her torso so that the tape was four layers thick (approximately 4 to $5 \mathrm{~mm}$ ) throughout. The body jacket was intimately molded over each subject's iliac crests and waist as a way of anchoring the orthosis and minimizing vertical migration on the trunk. The anterior, inferior trimline was located proximal to the inguinal fold, allowing the subject to flex the hip to $90^{\circ}$. The posterior, inferior trimline was located proximal to the sacrococcygeal junction and the posterior, superior trimline was located at the level of the inferior border of the axilla. The subject's "normal” postural alignment was not intentionally modified. The customized body jacket was removed from the subject by a posterior longitudinal cut along the axis of the spine and was further modified with holes (40 $\mathrm{mm}$ in diameter) through which the anterior superior iliac spine (ASIS) markers would protrude. Additionally, a posterior opening - typically used clinically - of $2.5 \mathrm{~cm}$ on either side of the spinous processes was cut to allow donning and doffing and to allow the sacral marker to remain in place between test conditions. Both gait evaluations were performed without alteration of any marker positions between the two data collection sessions. The posterior (a)

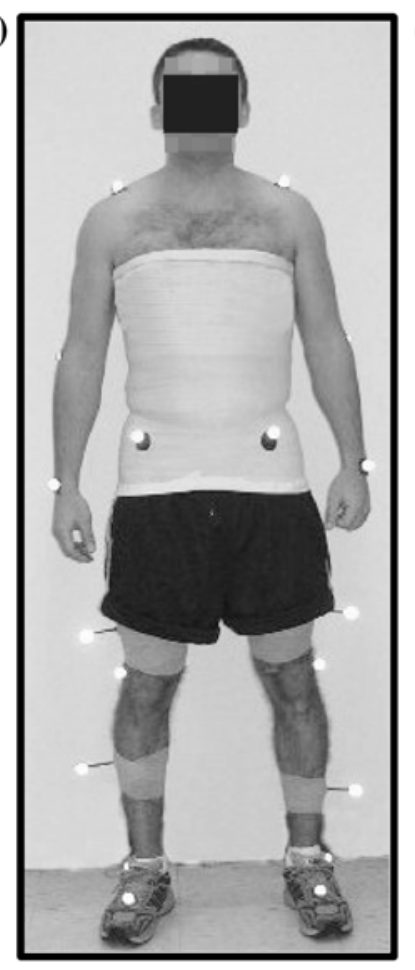

(b)

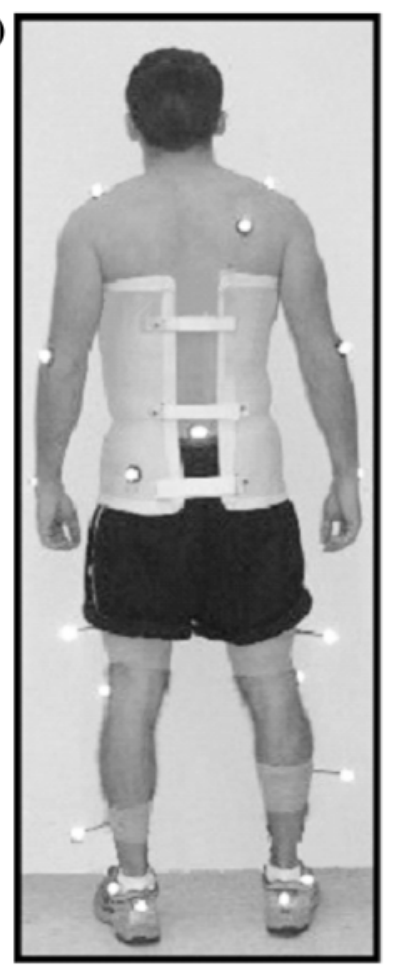

Figure 1.

(a) Anterior view of subject wearing customized fiberglass body jacket and retroreflective gait markers. Anterior pelvic markers were placed directly on skin over anterior superior iliac spines and protruded through holes in body jacket. (b) Posterior view. Posterior marker placed directly on body jacket was used for differentiating right and left sides of body and was not actually used for calculating kinematics.

opening was cinched by Velcro ${ }^{\mathrm{TM}}$ fabric hook-and-loop straps for a snug fit during gait. While the rigidity of this fixation was not directly quantified, routine clinical fitting procedures were used for assurance of a uniform snug fit. This orthotic design allowed for fabrication of a reasonably rigid customized body jacket at minimal expense and in a relatively short time periods, which enabled us to perform the unrestricted and restricted gait analyses in one testing session without moving any markers between conditions. The same orthotist finished fabricating the fiberglass body jacket by smoothing the edges, cutting holes, and attaching posterior straps while normal (unrestricted) gait data were collected from the subjects.

\section{Data Collection and Processing}

All motion data were collected in the Department of Veterans Affairs Chicago Motion Analysis Research Laboratory (VACMARL) (Chicago, Illinois), which was 
equipped with an eight-camera, Eagle Digital Real-Time motion-measurement system (Motion Analysis Corporation [MAC], Santa Rosa, California). We used this system to determine the instantaneous positions of the retroreflective markers on the body. We collected ground reaction force data simultaneously using six force platforms (Advanced Mechanical Technology, Inc, Watertown, Massachusetts) embedded flush with the walkway. Kinematic and kinetic data were acquired at $120 \mathrm{~Hz}$ and $960 \mathrm{~Hz}$, respectively. We used OrthoTrak software (MAC, Santa Rosa, California) to process the data. The subjects were asked to walk a minimum of five trials at each of five different self-selected walking speeds that were distributed across their comfortable range of speeds (normal, slow, very slow, fast, and very fast).

Following the first gait analysis, subjects donned the body jacket and we reevaluated their gait using the same protocol that was used for the unrestricted gait analysis. We fitted the body jacket to the subject without removing any markers and secured it with the posterior Velcro ${ }^{\mathrm{TM}}$ straps to ensure a snug fit. Data from the first gait analysis (unrestricted spinal motion) were analyzed and compared with the second gait analysis (restricted spinal motion).

\section{Data Analysis}

Temporospatial, kinematic, and kinetic data from the two gait analyses were compared at five self-selected walking speeds (normal, slow, very slow, fast, and very fast). Our goal was to record the patterns of motion across the subject's range of comfortable walking speeds and thereby analyze changes in gait parameters that vary as a function of speed. Three walking trials from each of the five selfselected walking speeds were chosen for analysis based on completeness of data; trials that had clean foot strikes on the force platforms as well as complete marker data were chosen. We averaged data from the three trials within each speed category to obtain a mean value for every subject for the parameters of interest (Table 2). These parameters were speed, step length, cadence, step width, single support time, double support time, pelvic obliquity, pelvic rotation, pelvic tilt, hip flexion-extension, hip abduction-adduction, hip rotation, knee flexion-extension during swing phase of the gait cycle, knee flexion-extension during stance phase, knee

Table 2.

Data (mean \pm standard deviation) from analyses of unrestricted $(U)$ versus restricted $(R)$ gait in 10 able-bodied subjects.

\begin{tabular}{|c|c|c|c|c|c|c|c|c|c|c|}
\hline \multirow{2}{*}{ Parameter } & \multicolumn{2}{|c|}{ Very Slow } & \multicolumn{2}{|c|}{ Slow } & \multicolumn{2}{|c|}{ Normal } & \multicolumn{2}{|c|}{ Fast } & \multicolumn{2}{|c|}{ Very Fast } \\
\hline & $\mathbf{U}$ & $\mathbf{R}$ & $\mathbf{U}$ & $\mathbf{R}$ & $\mathbf{U}$ & $\mathbf{R}$ & $\mathbf{U}$ & $\mathbf{R}$ & $\mathbf{U}$ & $\mathbf{R}$ \\
\hline Felocity (m/s) & $0.77 \pm 0.20$ & $0.86 \pm 0.25$ & $1.01 \pm 0.17$ & $1.12 \pm 0.24$ & $1.38 \pm 0.15$ & $1.36 \pm 0.33$ & $1.57 \pm 0.17$ & $1.53 \pm 0.39$ & $1.98 \pm 0.18$ & $81.88 \pm 0.18$ \\
\hline Tadence (steps/min) ${ }^{*}$ & $85.2 \pm 11.5$ & $90.0 \pm 10.1$ & $99.2 \pm 9.0$ & $102.7 \pm 7.3$ & $115.8 \pm 8.21$ & $117.3 \pm 6.3$ & $121.5 \pm 8.1$ & $124.9 \pm 7.6$ & $135.9 \pm 6.51$ & $139.7 \pm 7.7$ \\
\hline Step Width (cm) & $13.0 \pm 2.4$ & $12.8 \pm 2.9$ & $12.3 \pm 2.0$ & $12.8 \pm 2.5$ & $12.5 \pm 2.4$ & $12.6 \pm 2.1$ & $11.9 \pm 2.0$ & $12.2 \pm 1.9$ & $13.2 \pm 2.5$ & $12.6 \pm 2.1$ \\
\hline Single Support Time (\%) & $65.6 \pm 2.3$ & $65.2 \pm 1.6$ & $63.3 \pm 1.5$ & $63.4 \pm 1.4$ & $60.9 \pm 1.3$ & $61.1 \pm 1.6$ & $59.2 \pm 1.1$ & $59.3 \pm 1.6$ & $58.2 \pm 1.0$ & $58.2 \pm 1.6$ \\
\hline & $8.2 \pm 2.0$ & $7.3 \pm 2.1$ & $7.8 \pm 2.4$ & $6.7 \pm 1.8$ & $9.8 \pm 2.8$ & $7.8 \pm 2.0$ & $10.1 \pm 2.6$ & $7.4 \pm 2.3$ & $12.6 \pm 3.0$ & $9.2 \pm 2.5$ \\
\hline Pelvic Tilt $\left({ }^{\circ}\right)^{\dagger}$ & $2.0 \pm 0.4$ & $2.0 \pm 0.5$ & $2.0 \pm 0.5$ & $2.0 \pm 0.5$ & $2.3 \pm 0.8$ & $2.1 \pm 0.7$ & $2.2 \pm 0.8$ & $1.8 \pm 0.5$ & $2.9 \pm 1.1$ & $2.0 \pm 0.6$ \\
\hline Hip Flex-Ext $\left({ }^{\circ}\right)^{\ddagger}$ & $36.2 \pm 4.8$ & $38.0 \pm 5.9$ & $39.4 \pm 4.6$ & $41.4 \pm 4.9$ & $45.6 \pm 6.0$ & $45.1 \pm 5.0$ & $49.3 \pm 5.9$ & $49.9 \pm 5.8$ & $55.4 \pm 6.1$ & $54.2 \pm 5.4$ \\
\hline Hip Ab-Adduction $\left({ }^{\circ}\right)^{*}$ & $10.3 \pm 3.1$ & $7.3 \pm 2.1$ & $11.0 \pm 3.3$ & $7.7 \pm 2.1$ & $12.6 \pm 3.0$ & $8.2 \pm 2.2$ & $13.8 \pm 3.5$ & $8.9 \pm 2.3$ & $15.8 \pm 3.0$ & $9.7 \pm 2.4$ \\
\hline Ankle Flex-Ext $\left({ }^{\circ}\right)$ & $25.8 \pm 4.8$ & $25.5 \pm 5.2$ & $27.6 \pm 4.4$ & $28.6 \pm 5.2$ & $29.7 \pm 4.4$ & $29.8 \pm 4.2$ & $30.6 \pm 4.1$ & $31.2 \pm 4.6$ & $31.3 \pm 4.4$ & $31.2 \pm 5.0$ \\
\hline Ankle Rotation $\left({ }^{\circ}\right)$ & $16.5 \pm 4.6$ & $16.4 \pm 4.7$ & $17.2 \pm 4.6$ & $18.6 \pm 5.2$ & $19.3 \pm 5.0$ & $18.9 \pm 4.4$ & $20.8 \pm 5.8$ & $21.6 \pm 5.6$ & $21.4 \pm 6.1$ & $21.5 \pm 5.9$ \\
\hline VGRF (\% BW) & $1.01 \pm 0.02$ & $1.02 \pm 0.02$ & $1.03 \pm 0.04$ & $1.05 \pm 0.04$ & $1.18 \pm 0.07$ & $1.17 \pm 0.06$ & $1.26 \pm 0.10$ & $1.27 \pm 0.09$ & $1.46 \pm 0.09$ & $1.45 \pm 0.08$ \\
\hline 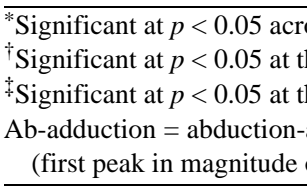 & and & & 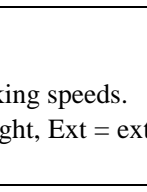 & 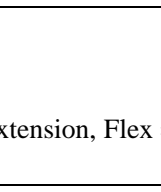 & & ( & & 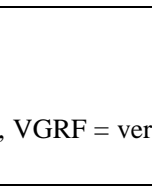 & & 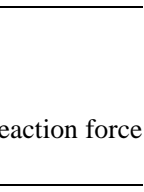 \\
\hline
\end{tabular}


rotation, ankle dorsiflexion-plantar flexion, ankle rotation, and the magnitude of the first peak in the VGRF. We used Shapiro-Wilk's normality test to ensure that the data were normally distributed [17]. We performed the same statistical analyses on all variables using a $5 \times 2$ two-way repeated measures analysis of variance (ANOVA) to compare data sets for five freely selected walking speeds (normal, slow, very slow, fast, and very fast) for the two spine-motion conditions (unrestricted and restricted) and for the interaction between speed and the spine-motion conditions. When interaction effects were significant at $p<0.05$, post hoc pairwise comparisons were made between unrestricted and restricted spinal conditions at each speed with the use of Bonferroni adjustments for multiple comparisons. We adjusted $t$-values using Statistical Package for the Social Sciences (SPSS ${ }^{\circledR}$ ) 11.0 software (SPSS, Inc, Chicago, Illinois) to reflect the Bonferroni correction; therefore, the $p$-value for the adjusted $t$-value is compared with 0.05 .

\section{RESULTS}

In this study, a $5 \times 2$ two-way repeated measures ANOVA was used for analysis of the 18 parameters across five self-selected walking speeds (normal, slow, very slow, fast, and very fast). For 12 of these parameters, restricted walking was not significantly different from unrestricted walking at $p<0.05$ (i.e., no significant main effects) and no significant interactions were found (Table 2). Since no main effects or interactions were significant, nothing more is reported for these parameters.

Pelvic obliquity range of motion (ROM) and hip abduction-adduction ROM showed significant main effects for restricted versus unrestricted walking ( $p<0.001$ ), as well as significant interactions $(p<0.001)$. Moreover, post hoc pairwise comparisons indicated significant differences for restricted versus unrestricted walking at each walking speed. For pelvic tilt ROM and hip flexion-extension ROM only significant interactions were observed ( $p=0.033$ and $p=0.013$, respectively) and post hoc pairwise comparisons were performed. Cadence and pelvic rotation ROM showed significant main effects for restriction ( $p=0.024$ and $p<$ 0.001 , respectively) but interactions were not significant. Results are summarized in Table 2.

Peak-to-peak (PP) pelvic rotation and obliquity motions were significantly reduced $(p<0.001)$ at all walking speeds when subjects walked with the body jacket (Figure 2(a) and 2(b)). Reduced PP pelvic tilt was also
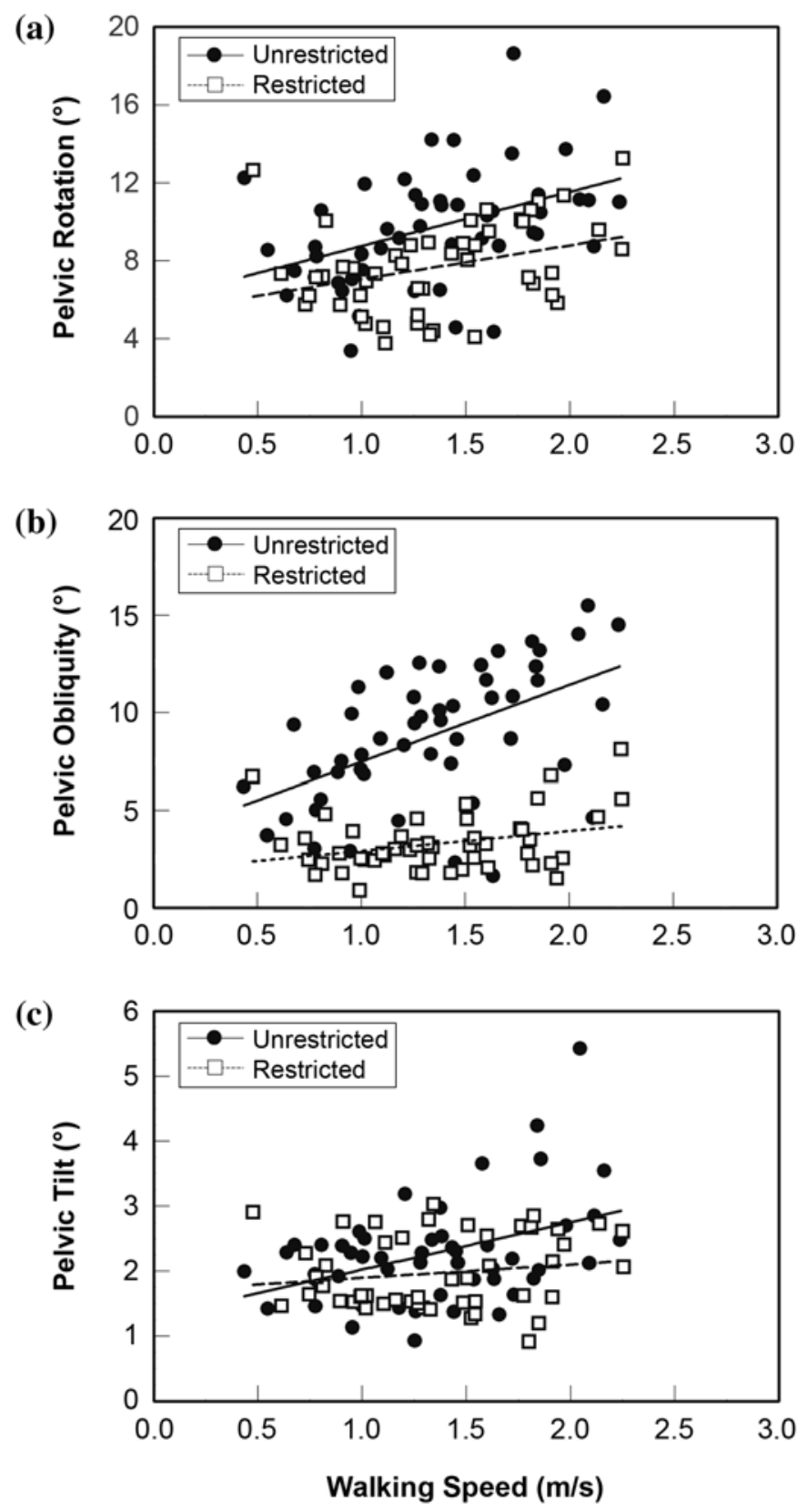

Figure 2.

(a) Peak-to-peak pelvic rotation (transverse plane), (b) pelvic obliquity (coronal plane), and (c) pelvic tilt (sagittal plane) for all subjects at all speeds with and without spinal restriction. Linear bestfit lines are shown for clarification of trends.

observed with spinal restriction across all walking speeds (Figure 2(c)) but was only significant at the fastest walking speeds $(p=0.017)$. Figure 3 depicts one subject's typical pelvic-motion curves with and without spinal restriction at the self-selected normal walking speed. 


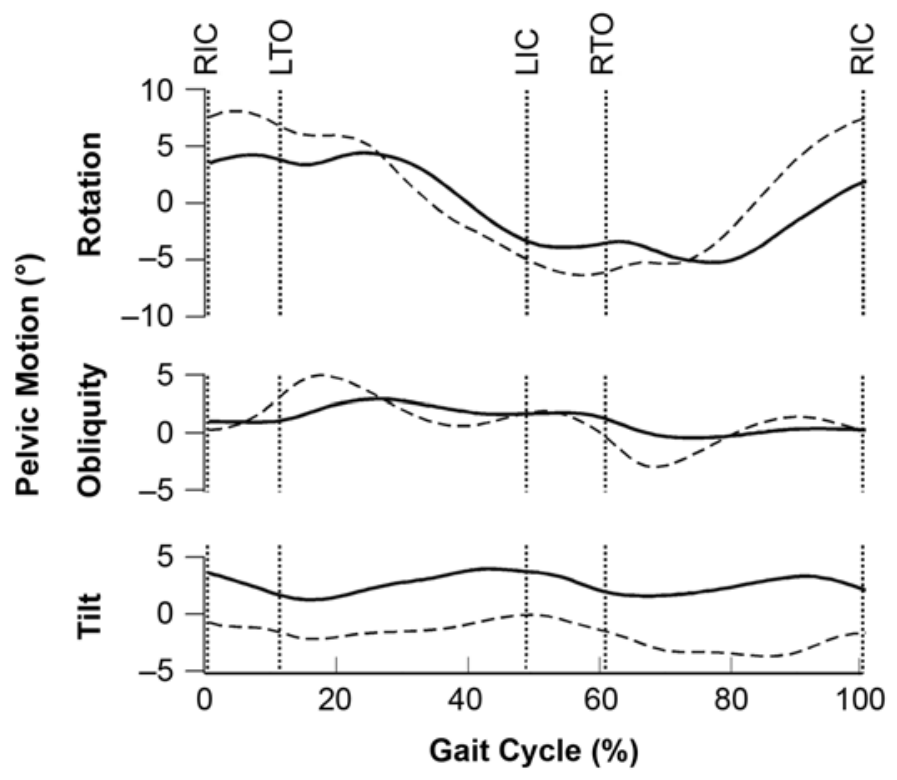

Figure 3.

One subject's typical pelvic motion curves for one walking trial at a self-selected normal speed, unrestricted (dashed line, $1.34 \mathrm{~m} / \mathrm{s}$ ) and restricted (solid line, $1.45 \mathrm{~m} / \mathrm{s}$ ). RIC = right initial contact, $\mathrm{LTO}=$ left toe off, LIC = left initial contact, RTO = right toe off.

No significant differences were observed in walking speeds between unrestricted and restricted spine conditions. However, analysis of the temporospatial parameters revealed that cadence, relative to each walking speed, was higher in the restricted condition than in the unrestricted condition ( $p=0.024$; Figure 4). While we observed a trend toward shorter step length with spinal restriction, step length did not significantly differ between the restricted and unrestricted conditions. Differences in step width were not significant, although larger step widths were observed at slower speeds in more than one-half of the subjects when walking with the body jacket. Additionally, no significant differences were found in single or double support times as a percentage of the gait cycle.

PP hip flexion-extension motion increased significantly with spinal restriction at the slow and very slow walking speeds ( $p<0.001$ and $p=0.023$, respectively). No significant differences were observed in PP knee or ankle sagittal plane motions between conditions, although a slight trend toward an increase in PP knee flexion during stance was observed across a range of selfselected walking speeds in the restricted condition. This same trend was also seen in PP swing-phase knee flexion at speeds below $1.8 \mathrm{~m} / \mathrm{s}$. PP hip abduction-adduction motion was significantly reduced with spinal restriction

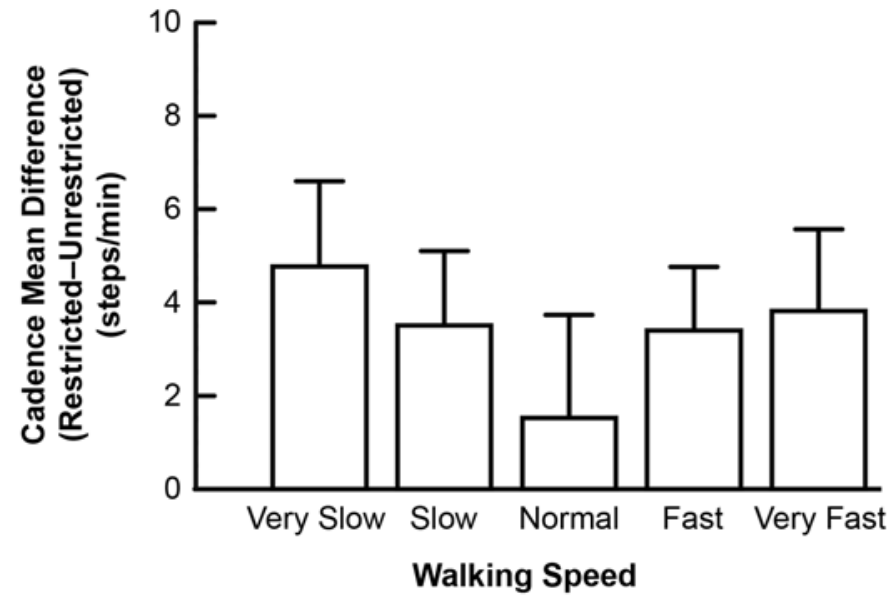

Figure 4.

Differences in mean group cadence (restricted vs unrestricted motion) and standard error of mean differences for each walking speed category for 10 able-bodied subjects.

across all speeds ( $p<0.001$; Figure 5). In the transverse plane, PP hip, knee, and ankle-joint rotations did not significantly differ between conditions. The magnitude of the first peak of the VGRF was unaffected by the body jacket and no significant differences were observed.

\section{DISCUSSION}

This study investigated the effect of spinal restriction on gait in able-bodied persons wearing a fiberglass body jacket. TLSOs that encompass the spine and the pelvis are designed to correct spinal alignment and/or provide stability, which involve restriction of spinal and pelvic motions. Consequently, spinal restriction with a TLSO may affect the contribution of spinal and pelvic motions to walking.

The results of this study demonstrated that when spinal motion was restricted, PP pelvic obliquity and rotation were significantly reduced across all walking speeds, while PP pelvic tilt was significantly reduced at only the fastest walking speeds. PP hip abduction-adduction ROM also significantly decreased during walking with spinal restriction. This reduction may have been directly associated with the reduction in PP pelvic obliquity since this measure represents relative coronal plane motion between the thigh and pelvis segments. No significant differences were observed in transverse-plane motions of the lowerlimb joints. However, PP hip flexion-extension significantly increased at the slow and slowest walking speeds, 


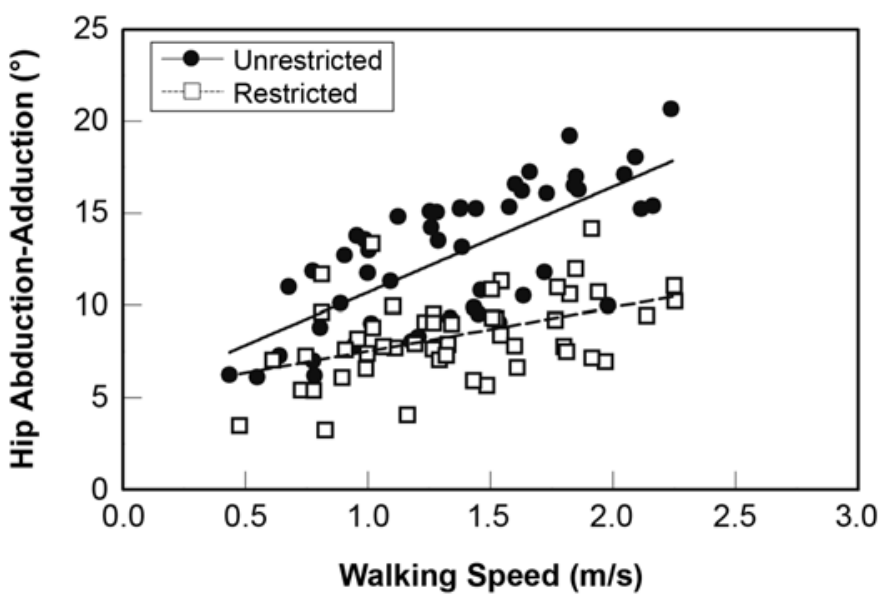

Figure 5.

Peak-to-peak hip abduction-adduction motion for 10 able-bodied subjects walking at a range of self-selected walking speeds with and without spinal restriction. Linear best-fit lines are shown for clarification of trends.

which possibly compensated for the loss in pelvic rotation that occurred with restriction. Interestingly, an offset in anterior pelvic tilt was noted for all subjects across all walking speeds with spinal restriction. This offset, seen in the restricted pelvic tilt curve (Figure 3), may have been unintentionally induced by the contouring of the body jacket over the iliac crests during casting of subjects in a standing position.

Walking speed and step length were expected to decrease significantly with spinal restriction, while cadence was expected to remain unchanged. However, these results were not observed. Cadence significantly increased with spinal restriction, while the other two temporospatial parameters were not significantly affected. Although not statistically significant, a trend toward shorter step lengths was observed in all individuals with spinal restriction and the significant reduction in PP pelvic rotation in the transverse plane possible explains this trend. The increased cadences may explain why walking speeds did not change significantly between conditions.

Step width was expected to increase to maintain adequate balance while subjects walked with a restricted spine. This result was not observed, which may indicate that balance was not substantially affected by spinal restriction during walking. Alternatively, the subjects' ability to achieve an increase in step width may have been limited since PP hip abduction-adduction ROM significantly decreased with spinal restriction and was possibly limited by the position of the lateral, inferior trimline of the body jacket.

Pelvic obliquity has been previously identified as a mechanism for shock attenuation during gait [13-14]. Previous research has also demonstrated that ankle plantar flexion and stance-phase knee flexion during the loading-response phase both decrease impact forces [13,1819]. We hypothesized that if pelvic obliquity were limited with spinal restriction, larger VGRFs or compensatory increases in sagittal plane motion at the ankle, knee, and/ or hip could occur. In this study, pelvic obliquity was reduced across all walking speeds with spinal restriction, which lessened the contribution of this motion to shock absorption during walking. However, the reduction in PP pelvic obliquity did not change the magnitude of the first peak of the VGRF between conditions. A trend toward increased PP stance-phase knee flexion was also observed in subjects walking with the body jacket, although this finding was not significant. This trend indicates that, when necessary, individuals may compensate for a loss of shock absorption associated with decreased pelvic obliquity by increasing their knee flexion during early stance phase. Compensation for reduced pelvic obliquity may also be possible with increased ankle plantar flexion, but this modification was not observed in this study. Increases in PP hip flexion-extension at walking speeds below $1.2 \mathrm{~m} / \mathrm{s}$ and small increases in stance-phase knee flexion across all walking speeds appeared to adequately absorb shock forces in able-bodied subjects because the VGRF magnitudes with and without spinal restriction were comparable.

Conventional polymer TLSOs are designed to be worn for relatively long time periods for correction or realignment of the spine in persons with a spinal pathology. However, the time and effort required for fabrication of polymer TLSOs were not practical for this study. Therefore, instead of a polymer TLSO, a fiberglass body jacket was used for restriction of spinal motion because its rigidity was deemed adequate for our purposes and because it was considered a practical alternative in terms of cost and ease of fabrication. Using a fiberglass body jacket allowed us to perform the unrestricted and restricted gait analyses in one testing session without moving any retroreflective skin-mounted markers between test conditions.

Some studies have indicated that spinal orthoses incompletely immobilize the lumbosacral junction. Miller et al. measured lumbosacral motion in and out of orthoses (lumbosacral corset, Jewett extension orthosis, and plastic TLSO) by using lateral flexion and extension roentgenograms in 
healthy individuals and in patients with spondylolisthesis [20]. For both subject groups, they reported a significant decrease in motion at the third to fourth lumbar (L3-L4) and L4-L5 spinal levels with the use of the Jewett orthosis and plastic TLSO $(p<0.01)$ but not at the L5 to first sacral (L5S1) spinal level. While our study did not look at spinal motion, the significantly reduced pelvic motions during walking with the body jacket suggest that more substantial restriction was achieved at the lumbo-sacral junction than was observed by Miller et al. [20]. This restriction may have resulted from the customization of the body jackets to each individual, the conformity of the orthosis over the iliac crests and waist, or the inferior trimline used. A tenet of orthotic clinical practice is that customized orthoses more effectively limit or control motion than prefabricated or off-the-shelf orthoses because better conformity of the orthosis to the patient may be achieved. Since this study did not involve intentional realignment of the spine or rectification of a positive plaster model, pelvic motion may not have been reduced as substantially as with a polymer TLSO vacuum-formed over a rectified model. With more substantial rectification over the iliac crests, waist, and abdomen and with better control of spinal alignment, pelvic motion during walking may be further reduced and the consequences for lower-limb kinematics and gait may be more substantial.

This study demonstrated that in able-bodied subjects, restriction of the spine with a customized fiberglass body jacket did have some effects on gait, particularly with regard to pelvic and hip ROM. While young, healthy, able-bodied subjects seemed to easily compensate for these effects, the clinical implications are that wearing a TLSO may have undesirable gait consequences in certain patient populations. For example, the increased hip flexion that was observed in response to diminished pelvic obliquity may be undesirable for patients with pathological conditions of the hip, such as osteoarthritis and/or weak hip musculature. While short-term effects may be minor, such as mild changes from "normal" gait, longterm effects may be more severe. Accelerated degenerative joint changes may result from compensatory motions and increased loading, both of which could potentially lead to pain and greater deviations from a normal gait pattern, although no data suggest that changes in gait represent a significant problem in people with limited spinal motion. Additionally, if lower-limb compensations are not possible or not of a sufficient magnitude for adequate shock attenuation, impact forces may increase and be transmitted to the spine and head. In persons who suffer from intervertebral disc degeneration, this may be of considerable concern. The patient may experience increased discomfort or pain, and shock forces that are felt at the head may affect vision and balance. A better understanding of the potential effects of spinal restriction on gait may help clinicians predict and avoid the development of additional problems that could result from restricting spinal motion with an orthosis. At the very least, an awareness of these issues will enable clinicians to monitor patients for problems that may result from walking with spinal orthoses.

The results of this study may also help explain why surgical fusions of the spine often lead to gait changes in patients with spinal pathologies such as scoliosis and lumbar flat back [21-22]. When the instrumentation for spinal fusion is anchored in the sacrum, it may have similar effects on pelvic motion as those observed in this current study of subjects walking with a restricted spine. However, the ability of people with spinal pathology to compensate for reduced pelvic and spinal motion may differ from that of the young, able-bodied subjects in this study.

This study has several limitations. The sample size was small, which makes generalizations difficult. Additionally, this research involved only young, healthy, able-bodied subjects for study of restricted spinal motion during gait, which is typically found in persons who have spinal pathology. We used able-bodied subjects to achieve a controlled, more uniform subject population than would be possible if this study had included subjects with spinal pathology. As a consequence, results at faster walking speeds in the ablebodied subjects may not directly relate to persons with spinal pathology who do not typically walk at these speeds. However, gait data at these faster walking speeds were collected because greater gait modifications are typically observed at the fastest speeds of both able-bodied subjects and subjects with spinal pathology compared with their freely-selected speeds, and this more dramatically illustrates the gait changes that may be associated with walking with restricted spinal motion.

The protocol described in this study may also be used for the study of spinal kinematics. The customized body jacket that was constructed allowed us to test multiple conditions without moving the gait analysis markers previously placed on the skin. In this study, holes were cut in the fiberglass body jacket over the two ASISs so that these markers could remain attached to the skin and protrude through the body jacket. The holes were $40 \mathrm{~mm}$ in diameter and located at least $22 \mathrm{~mm}$ from the inferior 
trimline, depending on the particular subject's anatomy. We believed that, for our purposes, the holes did not compromise the structural integrity and rigidity of the orthosis. A posterior opening, cinched by Velcro $^{\mathrm{TM}}$ straps, allowed the sacral marker to remain in place between test conditions. The posterior opening also permits the placement of additional spine markers if spinal kinematics are included in the gait assessment [23].

\section{CONCLUSIONS}

Our results indicated that able-bodied gait was nominally affected by spinal restriction. The customized fiberglass body jacket used in this study significantly reduced PP pelvic obliquity and rotation motion across all walking speeds and significantly reduced PP pelvic tilt at the fastest self-selected walking speed. It was not surprising that reductions in pelvic motion with spinal restriction also significantly affected lower-limb kinematics during gait since the pelvis forms a dynamic link between the lower limbs and trunk. Walking with the body jacket increased subjects' PP hip flexion-extension motion at the slow and very slow speeds while causing significantly reduced hip abduction-adduction motion across all walking speeds. No differences were observed in the peak magnitude of the VGRF with spinal restriction. These observations provide evidence of the able-bodied locomotor system's ability to adjust and adapt to reduced spinal motion to maintain balance, achieve stability, and possibly offset the greater shock forces that may be transmitted to the head during walking with a restricted spine. A better understanding of the effects of restricted spinal motion on gait may help clinicians predict and avoid additional problems that could result from TLSO use or surgical restriction of spinal motion. An awareness of these issues will enable clinicians to monitor patients for problems that may result from decreased spine and pelvic motion.

\section{ACKNOWLEDGMENTS}

Data for this project were collected at the VACMARL, Jesse Brown Department of Veterans Affairs Medical Center, Chicago, Illinois.

This material was based on work supported by the National Institutes of Health, grant 5T32HD07418-10, and the U.S. Department of Education National Institute on Disability and Rehabilitation Research, grants H133E980023 and H133E030030.

The opinions in this publication are those of the grantee and do not necessarily reflect those of the U.S. Department of Education.

The authors have declared that no conflicting interests exist.

\section{REFERENCES}

1. Thorstensson A, Nilsson J, Carlson H, Zomlefer MR. Trunk movements in human locomotion. Acta Physiol Scand. 1984;121(1):9-22. [PMID: 6741583]

2. Syczewska M, Oberg T, Karlsson D. Segmental movements of the spine during treadmill walking with normal speed. Clin Biomech (Bristol, Avon). 1999;14(6):384-88. [PMID: 10521619]

3. Feipel V, DeMesmaeker T, Klein P, Rooze M. Threedimensional kinematics of the lumbar spine during treadmill walking at different speeds. Eur Spine J. 2001;10(1): 16-22. [PMID: 11276830]

4. Smeathers JE. Transient vibrations caused by heel strike. Proc Inst Mech Eng [H]. 1989;203(4):181-86.

[PMID: 2701953

5. Brown T, Norton PL. The immobilizing efficiency of back braces; their effect on the posture and motion of the lumbosacral spine. J Bone Joint Surg Am. 1957;39A(1):111-39. [PMID: 13385269]

6. Fidler MW, Plasmans CM. The effect of four types of support on the segmental mobility of the lumbosacral spine. J Bone Joint Surg Am. 1983;65(7):943-47. [PMID: 6224799]

7. Tuong NH, Dansereau J, Maurais G, Herrera R. Threedimensional evaluation of lumbar orthosis effects on spinal behavior. J Rehabil Res Dev. 1998;35(1):34-42. [PMID: 9505251]

8. Lantz SA, Schultz AB. Lumbar spine orthosis wearing. I. Restriction of gross body motions. Spine. 1986;11(8):834-37. [PMID: 3810301]

9. Grew ND, Deane G. The physical effect of lumbar spinal supports. Prosthet Orthot Int. 1982;6(2):79-87. [PMID: 6213930]

10. Buchalter D, Kahanovitz N, Viola K, Dorsky S, Nordin M. Three-dimensional spinal motion measurements. Part 2: a noninvasive assessment of lumbar brace immobilization of the spine. J Spinal Disord. 1988;1(4):284-6.

[PMID: 2980256]

11. Waters RL, Morris JM. Effects of spinal supports on the electrical activity of muscles of the trunk. J Bone Joint Surg Am. 1970;52(1):51-60. [PMID: 4243832] 
12. Nachemson A, Elfstrom G. Intravital dynamic pressure measurements in the lumbar discs. A study of common movements, maneuvers and exercises. Scand J Rehabil Med Suppl. 1971;1:1-40. [PMID: 4257209]

13. Perry J. Gait analysis: normal and pathological function. Thorofare (NJ): Charles B. Slack, Inc; 1992.

14. Gard SA, Childress DS. The effect of pelvic list on the vertical displacement of the trunk during normal walking. Gait Posture. 1997;5(3):233-38.

15. Gard SA, Konz RJ. The effect of a shock-absorbing pylon on the gait of persons with unilateral transtibial amputation. J Rehabil Res Dev. 2003;40(2):109-24. [PMID: 15077637]

16. Kadaba MP, Ramakrishnan HK, Wootten ME. Measurement of lower extremity kinematics during level walking. J Orthop Res. 1990;8(3):383-92. [PMID: 2324857]

17. Field A. Discovering statistics using SPSS for Windows: advanced techniques for beginners. London (England): Sage Publications; 2000.

18. Nack JD, Phillips RD. Shock absorption. Clin Podiatr Med Surg. 1990;7(2):391-97. [PMID: 2189543]

19. Gard SA, Childress DS. The influence of stance-phase knee flexion on the vertical displacement of the trunk dur- ing normal walking. Arch Phys Med Rehabil. 1999;80(1): 26-32. [PMID: 9915368]

20. Miller RA, Hardcastle P, Renwick SE. Lower spinal mobility and external immobilization in the normal and pathologic condition. Orthop Rev. 1992;21(6):753-57. [PMID: 1614722]

21. Hasday CA, Passoff TL, Perry J. Gait abnormalities arising from latrogenic loss of lumbar lordosis secondary to Harrington instrumentation in lumbar fractures. Spine. 1983; 8(5):501-11. [PMID: 6648700]

22. Lee CS, Lee CK, Kim YT, Hong YM, Yoo JH. Dynamic sagittal imbalance of the spine in degenerative flat back. Spine. 2001;26(18):2029-35. [PMID: 11547204]

23. Konz RJ, Stine R, Ganju A, Fatone S, Jorge A, Gard SA, Childress DS, Ondra S. Development of an advanced biomechanical spine model to assess the effect of surgical stabilization and alignment on gait. In: Proceedings of the Congress of Neurological Surgeons 53rd Annual Meeting; 2003 Oct 18-23; Denver, Colorado. Schaumburg (IL): Congress of Neurological Surgeons; 2003.

Submitted for publication November 22, 2004. Accepted in revised form September 7, 2005. 